\title{
Absorbable Adhesion Barrier Gel
}

National Cancer Institute

\section{Source}

National Cancer Institute. Absorbable Adhesion Barrier Gel. NCI Thesaurus. Code C102848.

An isotonic, sterile, absorbable adhesion barrier gel composed of polyethylene oxide and sodium carboxymethylcellulose, with protective activity. Upon application of a single layer into the uterine cavity at the end of any hysteroscopic surgery, the absorbable adhesion barrier gel may provide a protective barrier which protects the traumatized tissue and allows for healing. This gel may therefore prevent the formation of post-surgical intrauterine adhesions. 